International Journal of Pure and Applied Mathematics

Volume 94 No. 4 2014, 501-513

ISSN: 1311-8080 (printed version); ISSN: 1314-3395 (on-line version)

url: http://www.ijpam.eu

doi: http://dx.doi.org/10.12732/ijpam.v94i4.5

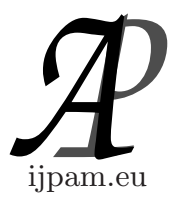

\title{
EXISTENCE OF WEAK SOLUTION FOR NONLINEAR ELLIPTIC SYSTEMS
}

\author{
Tahar Bouali ${ }^{1}$, Rafik Guefaifia ${ }^{2}$ \\ ${ }^{1,2}$ Department of Mathematics \\ Tebessa University \\ Tebessa, 12000, ALGERIA
}

Abstract: This paper is concerned with some nonlinear elliptic systems,Under suitable conditions on the nonlinearities $f$ and $g$, we obtain weak solution in sobolev space $H=H_{0}^{1}(\Omega) \times H_{0}^{1}(\Omega)$ by applying the Banach fixed point theorem.

AMS Subject Classification: 35D05, 35J60, 35J70, 15A18

Key Words: weak solution, nonlinear elliptic systems, Laplace operator, Riesz theorem, Banach fixed point theorem

\section{Introduction}

We study the nonlinear elliptic systems of the form

$$
\left\{\begin{array}{c}
-\Delta u-\operatorname{div}\left(\left(1+|\nabla u|^{2}\right)^{\frac{P(x)-2}{2}} \nabla u\right)=\lambda f(x, u, v) \text { in } \Omega \\
-\Delta v-\operatorname{div}\left(\left(1+|\nabla v|^{2}\right)^{\frac{P(x)-2}{2}} \nabla v\right)=\lambda g(x, u, v) \text { in } \Omega \\
u=v=0 \text { on } \partial \Omega
\end{array}\right.
$$

where $\Omega$ is a bounded smooth open set in $\mathbb{R}^{N}$. $(N \geq 3), p: \bar{\Omega} \rightarrow(1,2)$ is con-

Received: February 9, 2014

(C) 2014 Academic Publications, Ltd.

$\S$ Correspondence author url: www.acadpubl.eu 
tinuous function, $\lambda$ is positive constant and $\triangle u=\operatorname{div}(\nabla u)$ is the Laplacian of $u$.

Theorems concerning the existence and properties of fixed point are known as fixed point theorems.Such theorems are the most important tools for proving the existence and uniquencess of the solutions to various mathematical models (differential,integral and partial differential equation,ect...).

In recent years, many publications have appeared concerning quasilinear elliptic systems which have been used in a great variety of applications, we refer the readers to $[5,6,7,8,9]$ used variational methods to obtain weak solution of semlinear elliptic system and quasilinear elliptic system.

Motivated by [4] in this paper,we will discuss problem $(1,1)$, Under the suitable condition on the nonlinearities $f(x, u, v)$ and $g(x, u, v)$, using Banach fixed point theorem (see [10], we show that system $(1,1)$ has a unique weak solutions.

Throughout this paper for $(u, v) \in \mathbb{R}^{2}$, denote $|(u, v)|^{2}=|u|^{2}+|v|^{2}$. We assume that $f$ and $g$ are $L^{2}$ functions which are Lipschitz continuous with respect to the secand variable,i.e, there exist constants $c_{1}, c_{2} \succ 0$ such that for a.e. $x \in \Omega$ and for any $(u, v),\left(u_{1}, v_{1}\right) \in \mathbb{R}^{2}$

$$
\begin{aligned}
& \left|f(x, u, v)-f\left(x, u_{1}, v_{1}\right)\right| \leq c_{1}\left|(u, v)-\left(u_{1}, v_{1}\right)\right| \\
& \left|g(x, u, v)-g\left(x, u_{1}, v_{1}\right)\right| \leq c_{2}\left|(u, v)-\left(u_{1}, v_{1}\right)\right|
\end{aligned}
$$

Let $\lambda_{1}$ be the first eigenvalue of Dirichlet problem $-\Delta u=\lambda u$.The main result of this paper is a follows:

Definition 1. We say that $(u, v) \in H$ is a weak solution of $(1,1)$ if

$$
\begin{gathered}
\int_{\Omega}\left[\nabla u \nabla \xi+\nabla v \nabla \eta+\left(1+|\nabla u|^{2}\right)^{\frac{P(x)-2}{2}} \nabla u \nabla \xi+\left(1+|\nabla v|^{2}\right)^{\frac{P(x)-2}{2}} \nabla v \nabla \eta\right] d x \\
=\lambda \int_{\Omega}[f(x, u, v) \xi+g(x, u, v) \quad \eta] d x . \quad \text { for all }(\xi, \eta) \in H .
\end{gathered}
$$

Theorem 2. Suppose that conditions (1.2) and (1.3) hold. For any $\lambda \in$ $\left(0, \frac{\lambda_{1}}{c_{1}+c_{2}}\right)$ there existe a unique weak solution of $(1,1)$.

This paper is organized as follows. In Section 2, we present some relevant lemmas. We reserve the Section 3 for the proof of the main result. 


\section{Preliminary Lemmas}

Given a bounded smooth open set $\Omega \subset \mathbb{R}^{N}$. Let us consider the Hilbert space $H=H_{0}^{1}(\Omega) \times H_{0}^{1}(\Omega)$ and $\langle,\rangle_{L^{2}}$ the inner product in $L^{2}(\Omega)$. The norm on $H$ given by

$$
\|(u, v)\|=\left(\int_{\Omega}\left(|\nabla u|^{2}+|\nabla v|^{2}\right) d x\right)^{\frac{1}{2}}
$$

and the norm on $L^{2}(\Omega) \times L^{2}(\Omega)$ is given by

$$
\|(u, v)\|_{L^{2}(\Omega) \times L^{2}(\Omega)}=\left(\int_{\Omega}\left(|u|^{2}+|v|^{2}\right) d x\right)^{\frac{1}{2}}
$$

Lemma 3. Let $F: \Omega \times \mathbb{R}^{N} \rightarrow \mathbb{R}$ be on operator defined by

$$
F(x, \xi)=\left(1+|\xi|^{2}\right)^{\frac{P(x)-2}{2}}, \forall x \in \Omega, \forall \xi \in \mathbb{R}^{N}
$$

then

$$
F(x, \xi) \leq 1
$$

for all $\xi \in \mathbb{R}^{N}$ and all $x \in \Omega$.

Proof. We fix $x_{0} \in \bar{\Omega}$ and denote by $q=p\left(x_{0}\right)$.Then

$$
F\left(x_{0}, \xi\right)=\frac{1}{\left(1+|\xi|^{2}\right)^{\frac{2-q}{2}}}
$$

where

$$
0<\frac{2-q}{2}<1
$$

We define the auxiliary function $g:[0, \infty) \rightarrow \mathbb{R}$ by

$$
g(t)=\left(1+t^{2}\right)^{\frac{2-q}{2}}
$$

It can be easily seen that $g$ is increasing on $[0, \infty)$ since $\frac{2-q}{2}>0$.Thus, we obtain that $g(t) \geq g(0)=1, \forall t \geq 0$. Taking $t=|\xi|$, the conclusion of the lemma follows.

Lemma 4. For any $\xi, \eta \in \mathbb{R}^{N}$, any $x \in \bar{\Omega}$ and any $i \in\{1,2, \ldots, N\}$ the following inequalities hold true: 


$$
\begin{gathered}
{\left[\left(1+|\xi|^{2}\right)^{\frac{P(x)-2}{2}} \xi-\left(1+|\eta|^{2}\right)^{\frac{P(x)-2}{2}} \eta\right] \cdot(\xi-\eta) \geq 0 .} \\
{\left[\left(1+|\xi|^{2}\right)^{\frac{P(x)-2}{2}} \xi_{i}-\left(1+|\eta|^{2}\right)^{\frac{P(x)-2}{2}} \eta_{i}\right] \leq \frac{N+3}{2}|\xi-\eta| .}
\end{gathered}
$$

Here, the symbol "." means the inner product in $\mathbb{R}^{N}$.

Proof. (a) Let $x_{0} \in \Omega$ be arbitrary but fixed, and define $q=p\left(x_{0}\right) \in$ $(1,2)$. Our inequality is equivalent with

$$
\left[\left(1+|\xi|^{2}\right)^{\frac{q-2}{2}}|\xi|^{2}+\left(1+|\eta|^{2}\right)^{\frac{q-2}{2}}|\eta|^{2}\right] \geq\left[\left(1+|\xi|^{2}\right)^{\frac{q-2}{2}}+\left(1+|\eta|^{2}\right)^{\frac{q-2}{2}}\right] \xi . \eta .
$$

We shall prove that

$$
\left(1+|\xi|^{2}\right)^{\frac{q-2}{2}}|\xi|^{2}+\left(1+|\eta|^{2}\right)^{\frac{q-2}{2}}|\eta|^{2} \geq\left[\left(1+|\xi|^{2}\right)^{\frac{q-2}{2}}+\left(1+|\eta|^{2}\right)^{\frac{q-2}{2}}\right]|\xi \cdot \eta|
$$

or

$$
\left[\left(1+|\xi|^{2}\right)^{\frac{q-2}{2}}|\xi|-\left(1+|\eta|^{2}\right)^{\frac{q-2}{2}}|\eta|\right](|\xi|-|\eta|) \geq 0
$$

We define the auxiliary function $\phi:[0, \infty) \rightarrow \mathbb{R}$ by $\phi(t)=\left(1+t^{2}\right)^{\frac{q-2}{2}} t$. Obviously $\phi$ is derivable and we have:

$$
\phi^{\prime}(t)=\left(1+t^{2}\right)^{\frac{q-4}{2}}\left[1+(q-1) t^{2}\right] \geq 0
$$

Hence $\phi$ is increasing on $[0, \infty)$ which implies

$$
\left[\phi\left(t_{1}\right)-\phi\left(t_{2}\right)\right]\left(t_{1}-t_{2}\right) \geq 0, \forall t_{1}, t_{2} \in[0, \infty] .
$$

Taking $t_{1}=|\xi|, t_{2}=|\eta|$ the inequality (1.1) follows.

(b) Let $x \in \bar{\Omega}$ be fixed and $h_{i}: \mathbb{R}^{N} \rightarrow \mathbb{R}$ be defined by

$h_{i}(\xi)=\left(1+|\xi|^{2}\right)^{\frac{P(x)-2}{2}} \xi_{i}, \forall \xi \in \mathbb{R}^{N}, \forall i \in\{1,2, \ldots, N\}$.

Using the mean value theorem we deduce that

$$
\left|h_{i}(\xi)-h_{i}(\eta)\right| \leq|\xi-\eta| \sup _{\theta \in[\xi, \eta]}\left|\nabla h_{i}(\theta)\right|
$$

where $[\xi, \eta]$ is the line segment in $\mathbb{R}^{N}$ between the points $\xi$ and $\eta$, i.e.,

$$
[\xi, \eta]=\{t \xi+(1-t) \eta: t \in[0,1]\}
$$


but

$$
\left|\nabla h_{i}(\theta)\right|=\left(\sum_{j=1}^{N}\left(\frac{\partial h_{i}(\theta)}{\partial \theta_{j}}\right)^{2}\right)^{\frac{1}{2}} \leq \sum_{j=1}^{N}\left|\frac{\partial h_{i}(\theta)}{\partial \theta_{j}}\right|
$$

For $j \neq i$

$$
\frac{\partial h_{i}(\theta)}{\partial \theta_{j}}=(p(x)-2)\left(1+|\theta|^{2}\right)^{\frac{P(x)-2}{2}} \theta_{i} \theta_{j}
$$

and for $j=i$

$$
\frac{\partial h_{i}(\theta)}{\partial \theta_{j}}=\left(1+|\theta|^{2}\right)^{\frac{P(x)-2}{2}}+(p(x)-2)\left(1+|\theta|^{2}\right)^{\frac{P(x)-2}{2}} \theta_{i}^{2} .
$$

Thus, by $(2,3)$ and lemma 3 we find

$$
\begin{gathered}
\left|\nabla h_{i}(\theta)\right| \leq \sum_{j=1}^{N}\left|\frac{\partial h_{i}(\theta)}{\partial \theta_{j}}\right| \\
\leq\left(1+|\theta|^{2}\right)^{\frac{P(x)-2}{2}}+\sum_{j=1}^{N}\left|(p(x)-2)\left(1+|\theta|^{2}\right)^{\frac{p(x)-4}{2}} \theta_{i} \theta_{j}\right| \\
=\left(1+|\theta|^{2}\right)^{\frac{P(x)-2}{2}}+(2-p(x))\left(1+|\theta|^{2}\right)^{\frac{p(x)-4}{2}} \sum_{j=1}^{N}\left|\theta_{i} \theta_{j}\right| \\
\leq 1+(2-p(x))\left(1+|\theta|^{2}\right)^{\frac{p(x)-4}{2}} \sum_{j=1}^{N} \frac{\theta_{i}^{2}+\theta_{j}^{2}}{2} \\
\leq 1+(2-p(x))\left(1+|\theta|^{2}\right)^{\frac{p(x)-4}{2}} \frac{N+1}{2}|\theta|^{2} \\
\leq 1+(2-p(x))\left(1+|\theta|^{2}\right)^{\frac{p(x)-2}{2}} \frac{N+1}{2} \\
\leq 1+\frac{N+1}{2}=\frac{N+3}{2}
\end{gathered}
$$

Combining the above estimates with relation (2.2) we obtain

$$
\left|\left(1+|\xi|^{2}\right)^{\frac{P(x)-2}{2}} \xi_{i}-\left(1+|\eta|^{2}\right)^{\frac{P(x)-2}{2}} \eta_{i}\right| \leq \frac{N+3}{2}|\xi-\eta| .
$$

The proof of lemma 4 is now complet.

Proof of the main result. In order to prove theorem 2 we use a methode borrowed from the proof of a nonlinear version of the Lax-Milgram theorem

(see Zeidler [10], section 2.15) .Our proof will use as main tool the Banach fixed point theorem (see Zeidler [10], section 1.6) 
First,we define the operators $a: H \times H \rightarrow \mathbb{R}$ by

$$
\begin{aligned}
a((u, v),(\xi, \eta))= & \int_{\Omega} \nabla u \nabla \xi d x+\int_{\Omega} \nabla v \nabla \eta d x+\int_{\Omega}\left(1+|\nabla u|^{2}\right)^{\frac{P(x)-2}{2}} \nabla u \nabla \xi d x \\
& +\int_{\Omega}\left(1+|\nabla v|^{2}\right)^{\frac{P(x)-2}{2}} \nabla v \nabla \eta d x,
\end{aligned}
$$

respectively $b_{\lambda}: H \times H \rightarrow \mathbb{R}$ by

$$
b_{\lambda}((u, v),(\xi, \eta))=\lambda\left[\int_{\Omega} f(x, u, v) \xi d x+\int_{\Omega} g(x, u, v) \eta d x\right\rfloor .
$$

Lemma 5. The operators $a$ and $b_{\lambda}$ satisfy the following properties:

$\left(A_{1}\right)$ for each $(u, v) \in H$, the application $(\xi, \eta) \longmapsto a((u, v),(\xi, \eta))$ is linear and continuous.

$\left(A_{2}\right)$

$$
a\left((u, v),(u, v)-\left(u_{1}, v_{1}\right)\right)-a\left(\left(u_{1}, v_{1}\right),(u, v)-\left(u_{1}, v_{1}\right)\right) \geq\left\|(u, v)-\left(u_{1}, v_{1}\right)\right\|^{2}
$$

for all $(u, v),\left(u_{1}, v_{1}\right) \in H$.

$\left(A_{3}\right)$ there exist $M>0$ such that

$$
\left|a((u, v),(\xi, \eta))-a\left(\left(u_{1}, v_{1}\right),(\xi, \eta)\right)\right| \leq M\left\|(u, v)-\left(u_{1}, v_{1}\right)\right\|\|(\xi, \eta)\|
$$

for all $(u, v),\left(u_{1}, v_{1}\right),(\xi, \eta) \in H$.

$\left(B_{1}\right)$ for each $(u, v) \in H$, the application $(\xi, \eta) \longmapsto b_{\lambda}((u, v),(\xi, \eta))$ is linear and continuous.

$\left(B_{2}\right)$

$$
\begin{gathered}
b_{\lambda}\left((u, v),(u, v)-\left(u_{1}, v_{1}\right)\right)-b_{\lambda}\left(\left(u_{1}, v_{1}\right),(u, v)-\left(u_{1}, v_{1}\right)\right) \\
\leq \frac{\lambda\left(c_{1}+c_{2}\right)}{\lambda_{1}}\left\|(u, v)-\left(u_{1}, v_{1}\right)\right\|^{2}
\end{gathered}
$$

for all $(u, v),\left(u_{1}, v_{1}\right) \in H .\left(B_{3}\right)$ there exist $N=N(\lambda)>0$ such that

$$
\left|b_{\lambda}((u, v),(\xi, \eta))-b_{\lambda}\left(\left(u_{1}, v_{1}\right),(\xi, \eta)\right)\right| \leq N\left\|(u, v)-\left(u_{1}, v_{1}\right)\right\|\|(\xi, \eta)\|
$$

for all $(u, v),\left(u_{1}, v_{1}\right),(\xi, \eta) \in H$. 
Proof. $\left(A_{1}\right)$ We fixe $(u, v) \in H$. It is clear that $(\xi, \eta) \longmapsto a((u, v),(\xi, \eta))$ is linear. On the other hand, using Lemma 3 and Holder's inequality we have

$$
\begin{aligned}
|a((u, v),(\xi, \eta))|= & \mid \int_{\Omega} \nabla u \nabla \xi d x+\int_{\Omega} \nabla v \nabla \eta d x+\int_{\Omega}\left(1+|\nabla u|^{2}\right)^{\frac{P(x)-2}{2}} \nabla u \nabla \xi d x \\
& +\int_{\Omega}\left(1+|\nabla v|^{2}\right)^{\frac{P(x)-2}{2}} \nabla v \nabla \eta d x \mid \leq 4\|(u, v)\|\|(\xi, \eta)\|
\end{aligned}
$$

it follows that $(\xi, \eta) \longmapsto a((u, v),(\xi, \eta))$ is continuous.

$\left(A_{2}\right)$ Using Lemma 4, part $(a)$, we deduce that

$$
\begin{aligned}
& a\left((u, v),(u, v)-\left(u_{1}, v_{1}\right)\right)-a\left(\left(u_{1}, v_{1}\right),(u, v)-\left(u_{1}, v_{1}\right)\right) \\
& =\left\|(u, v)-\left(u_{1}, v_{1}\right)\right\|^{2} \\
& +\int_{\Omega}\left(1+|\nabla u|^{2}\right)^{\frac{P(x)-2}{2}}|\nabla u|^{2}+\left(1+\left|\nabla u_{1}\right|^{2}\right)^{\frac{P(x)-2}{2}}\left|\nabla u_{1}\right|^{2} \\
& \left.-\left(1+|\nabla u|^{2}\right)^{\frac{P(x)-2}{2}} \nabla u \nabla u_{1}-\left(1+\left|\nabla u_{1}\right|^{2}\right)^{\frac{P(x)-2}{2}} \nabla u \nabla u_{1}\right) d x \\
& +\int_{\Omega}\left(1+|\nabla v|^{2}\right)^{\frac{P(x)-2}{2}}|\nabla v|^{2}+\left(1+\left|\nabla v_{1}\right|^{2}\right)^{\frac{P(x)-2}{2}}\left|\nabla v_{1}\right|^{2} \\
& \left.-\left(1+|\nabla v|^{2}\right)^{\frac{P(x)-2}{2}} \nabla v \nabla v_{1}-\left(1+\left|\nabla v_{1}\right|^{2}\right)^{\frac{P(x)-2}{2}} \nabla v \nabla v_{1}\right) d x \\
& =\left\|(u, v)-\left(u_{1}, v_{1}\right)\right\|^{2} \\
& +\int_{\Omega}\left[\left(1+|\nabla u|^{2}\right)^{\frac{P(x)-2}{2}} \nabla u-\left(1+\left|\nabla u_{1}\right|^{2}\right)^{\frac{P(x)-2}{2}} \nabla u_{1}\right] \cdot\left(\nabla u-\nabla u_{1}\right) d x \\
& +\int_{\Omega}\left[\left(1+|\nabla v|^{2}\right)^{\frac{P(x)-2}{2}} \nabla v-\left(1+\left|\nabla v_{1}\right|^{2}\right)^{\frac{P(x)-2}{2}} \nabla v_{1}\right] \cdot\left(\nabla v-\nabla v_{1}\right) d x \\
& \geq\left\|(u, v)-\left(u_{1}, v_{1}\right)\right\|^{2}
\end{aligned}
$$

$\left(A_{3}\right)$ Using Holder's inequality and Lemma 4 part $(b)$ we get

$$
\begin{aligned}
& \left|a((u, v),(\xi, \eta))-a\left(\left(u_{1}, v_{1}\right),(\xi, \eta)\right)\right| \\
= & \int_{\Omega}\left(\nabla u-\nabla u_{1}\right) \nabla \xi d x+\int_{\Omega}\left(\nabla v-\nabla v_{1}\right) \nabla \eta d x
\end{aligned}
$$




$$
\begin{aligned}
& +\int_{\Omega}\left[\left(1+|\nabla u|^{2}\right)^{\frac{P(x)-2}{2}} \nabla u-\left(1+\left|\nabla u_{1}\right|^{2}\right)^{\frac{P(x)-2}{2}} \nabla u_{1}\right] \nabla \xi d x \\
& +\int_{\Omega}\left[\left(1+|\nabla v|^{2}\right)^{\frac{P(x)-2}{2}} \nabla v-\left(1+\left|\nabla v_{1}\right|^{2}\right)^{\frac{P(x)-2}{2}} \nabla v_{1}\right] \nabla \eta d x \\
& \leq 2\left\|(u, v)-\left(u_{1}, v_{1}\right)\right\|\|(\xi, \eta)\| \\
& +\int_{\Omega} \sum_{j=1}^{N}\left|\left(1+|\nabla u|^{2}\right)^{\frac{P(x)-2}{2}} \frac{\partial u}{\partial x_{j}}-\left(1+\left|\nabla u_{1}\right|^{2}\right)^{\frac{P(x)-2}{2}} \frac{\partial u_{1}}{\partial x_{j}}\right|\left|\frac{\partial \xi}{\partial x_{j}}\right| d x \\
& +\int_{\Omega} \sum_{j=1}^{N}\left|\left(1+|\nabla v|^{2}\right)^{\frac{P(x)-2}{2}} \frac{\partial v}{\partial x_{j}}-\left(1+\left|\nabla v_{1}\right|^{2}\right)^{\frac{P(x)-2}{2}} \frac{\partial v_{1}}{\partial x_{j}}\right|\left|\frac{\partial \eta}{\partial x_{j}}\right| d x \\
& \leq 2\left\|(u, v)-\left(u_{1}, v_{1}\right)\right\|\|(\xi, \eta)\|+\frac{N+3}{2} \int_{\Omega} \sum_{j=1}^{N}\left|\left(\nabla u-\nabla u_{1}\right)\right|\left|\frac{\partial \xi}{\partial x_{j}}\right| d x \\
& +\frac{N+3}{2} \int_{\Omega} \sum_{j=1}^{N}\left|\left(\nabla v-\nabla v_{1}\right)\right|\left|\frac{\partial \eta}{\partial x_{j}}\right| d x \\
& \leq 2\left\|(u, v)-\left(u_{1}, v_{1}\right)\right\|\|(\xi, \eta)\|+\frac{N+3}{2} \int_{\Omega}\left|\left(\nabla u-\nabla u_{1}\right)\right| \sum_{j=1}^{N}\left|\frac{\partial \xi}{\partial x_{j}}\right| d x \\
& +\frac{N+3}{2} \int_{\Omega}\left|\left(\nabla v-\nabla v_{1}\right)\right| \sum_{j=1}^{N}\left|\frac{\partial \eta}{\partial x_{j}}\right| d x \\
& \leq 2\left\|(u, v)-\left(u_{1}, v_{1}\right)\right\|\|(\xi, \eta)\|+(N+3) \sqrt{N}\left\|(u, v)-\left(u_{1}, v_{1}\right)\right\|\|(\xi, \eta)\| \\
& \leq(2+(N+3) \sqrt{N})\left\|(u, v)-\left(u_{1}, v_{1}\right)\right\|\|(\xi, \eta)\|=M\left\|(u, v)-\left(u_{1}, v_{1}\right)\right\|\|(\xi, \eta)\|
\end{aligned}
$$

where $M=2+(N+3) \sqrt{N}$.

$\left(B_{1}\right)$ We fixe $(u, v) \in H$.obviously, the application $(\xi, \eta) \longmapsto b_{\lambda}((u, v),(\xi, \eta))$ is linear.

Using Holder's inequality, we have

$$
\left|b_{\lambda}((u, v),(\xi, \eta))\right|=\left|\lambda\left[\int_{\Omega} f(x, u, v) \xi d x+\int_{\Omega} g(x, u, v) \eta \mathbf{d x}\right]\right|
$$




$$
\begin{aligned}
& \leq \lambda\left(\int_{\Omega}|f(x, u, v)|^{2} d x\right)^{\frac{1}{2}}\left(\int_{\Omega}|\xi|^{2} d x\right)^{\frac{1}{2}} \\
& +\lambda\left(\int_{\Omega}|g(x, u, v)|^{2} d x\right)^{\frac{1}{2}}\left(\int_{\Omega}|\eta|^{2} d x\right)^{\frac{1}{2}} \leq C\|(\xi, \eta)\|,
\end{aligned}
$$

where $C$ is positive constant.

$\left(B_{2}\right)$

$$
\begin{aligned}
& b_{\lambda}\left((u, v),(u, v)-\left(u_{1}, v_{1}\right)\right)-b_{\lambda}\left(\left(u_{1}, v_{1}\right),(u, v)-\left(u_{1}, v_{1}\right)\right) \\
= & \lambda \int_{\Omega}\left[f(x, u, v)-f\left(x, u_{1}, v_{1}\right)\right]\left(u-u_{1}\right) d x \\
& +\lambda \int_{\Omega}\left[g(x, u, v)-g\left(x, u_{1}, v_{1}\right)\right]\left(v-v_{1}\right) d x \\
\leq & \left.\left.\lambda c_{1}\left(\int_{\Omega}\left|u-u_{1}\right|^{2}+\left|v-v_{1}\right|^{2}\right)^{\frac{1}{2}}\right)_{\Omega} \int_{\Omega}\left|u-u_{1}\right|^{2} d x\right)^{\frac{1}{2}}\left(\int_{\Omega}\left|v-v_{1}\right|^{2} d x\right. \\
& +\lambda c_{2}\left(\int_{\Omega}\left|u-u_{1}\right|^{2}+\left|v-v_{1}\right|^{2} d x\right. \\
\leq & \lambda c_{1}\left\|(u, v)-\left(u_{1}, v_{1}\right)\right\|_{L^{2}(\Omega) \times L^{2}(\Omega)}^{2}+\lambda c_{2}\left\|(u, v)-\left(u_{1}, v_{1}\right)\right\|_{L^{2}(\Omega) \times L^{2}(\Omega)}^{2} \\
\leq & \lambda\left(c_{1}+c_{2}\right)\left\|(u, v)-\left(u_{1}, v_{1}\right)\right\|_{L^{2}(\Omega) \times L^{2}(\Omega)}^{2} \\
\leq & \frac{\lambda\left(c_{1}+c_{2}\right)}{\lambda_{1}}\left\|(u, v)-\left(u_{1}, v_{1}\right)\right\|^{2} .
\end{aligned}
$$

$\left(B_{3}\right)$ Using Holder's inequality and $(1.2),(1.3)$ we obtain

$$
\begin{aligned}
& \left|b_{\lambda}((u, v),(\xi, \eta))-b_{\lambda}\left(\left(u_{1}, v_{1}\right),(\xi, \eta)\right)\right| \\
= & \left|\lambda \int_{\Omega}\left[f(x, u, v)-f\left(x, u_{1}, v_{1}\right)\right] \xi d x+\lambda \int_{\Omega}\left[g(x, u, v)-g\left(x, u_{1}, v_{1}\right)\right] \eta d x\right| \\
\leq & \lambda c_{1}\left\|(u, v)-\left(u_{1}, v_{1}\right)\right\|_{L^{2}(\Omega) \times L^{2}(\Omega)}^{2}\left(\int_{\Omega}|\xi|^{2} d x\right)
\end{aligned}
$$




$$
\begin{aligned}
& +\lambda c_{2}\left\|(u, v)-\left(u_{1}, v_{1}\right)\right\|_{L^{2}(\Omega) \times L^{2}(\Omega)}^{2}\left(\int_{\Omega}|\eta|^{2} d x\right) \\
\leq & \lambda\left(c_{1}+c_{2}\right)\left\|(u, v)-\left(u_{1}, v_{1}\right)\right\|_{L^{2}(\Omega) \times L^{2}(\Omega)}^{2}\|(\xi, \eta)\|_{L^{2}(\Omega) \times L^{2}(\Omega)} \\
\leq & \frac{\lambda\left(c_{1}+c_{2}\right)}{\lambda_{1}}\left\|(u, v)-\left(u_{1}, v_{1}\right)\right\|\|(\xi, \eta)\| \\
\leq & N\left\|(u, v)-\left(u_{1}, v_{1}\right)\right\|\|(\xi, \eta)\|,
\end{aligned}
$$

where $N=\frac{\lambda\left(c_{1}+c_{2}\right)}{\lambda_{1}}$.

\section{Proof of Main Theorem}

In this section we give the proof of theorem 2 .

Proof of Theorem 2. Let $\lambda \in\left(0, \frac{\lambda_{1}}{c_{1}+c_{2}}\right)$ be arbitrary but fixed.By lemma 5. $\left(A_{1}\right)$ and the Riesz theorem (see e.g. Brezis [2], theorem V.5) we

deduce that for each $(u, v) \in H$ there existe a unique element denote by $A(u, v) \in H$ such that $a((u, v),(\xi, \eta))=\langle A(u, v),(\xi, \eta)\rangle$.

Thus we can define the operator $A: H \rightarrow H$.Using lemma 5. $\left(A_{2}\right)$ it follows that

$$
\left\langle A(u, v)-A\left(u_{1}, v_{1}\right),(u, v)-\left(u_{1}, v_{1}\right)\right\rangle \geq\left\|(u, v)-\left(u_{1}, v_{1}\right)\right\|^{2}
$$

for all $(u, v),\left(u_{1}, v_{1}\right) \in H$ i.e, $A$ is strongly monotone.

lemma 5. $\left(A_{3}\right)$ yields

$$
\left|\langle A(u, v),(\xi, \eta)\rangle-\left\langle A\left(u_{1}, v_{1}\right),(\xi, \eta)\right\rangle\right| \leq M\left\|(u, v)-\left(u_{1}, v_{1}\right)\right\|\|(\xi, \eta)\|
$$

for all $(u, v),\left(u_{1}, v_{1}\right),(\xi, \eta) \in H$.Hence,

$$
\left\|A(u, v)-A\left(u_{1}, v_{1}\right)\right\|=\sup _{\|(\xi, \eta)\| \leq 1}\left|A\left(u_{1}, v_{1}\right),(\xi, \eta)\right| \leq M\left\|(u, v)-\left(u_{1}, v_{1}\right)\right\|
$$

i.e, $A$ is Lipschitz continuous.

By lemma 5. $\left(B_{1}\right)$ and the Riesz theorem we deduce that for each $(u, v) \in H$ there exists a unique element $B_{\lambda}(u, v) \in H$ such that

$$
b_{\lambda}((u, v),(\xi, \eta))=\left\langle B_{\lambda}(u, v),(\xi, \eta)\right\rangle, \forall(\xi, \eta) \in H .
$$


Thus, we can also define the operator $B_{\lambda}: H \rightarrow H$ which satisfies

$$
\begin{gathered}
\left\langle B_{\lambda}(u, v),(u, v)-\left(u_{1}, v_{1}\right)\right\rangle-\left\langle B_{\lambda}\left(u_{1}, v_{1}\right),(u, v)-\left(u_{1}, v_{1}\right)\right\rangle \\
\leq \frac{\lambda\left(c_{1}+c_{2}\right)}{\lambda_{1}}\left\|(u, v)-\left(u_{1}, v_{1}\right)\right\|^{2}
\end{gathered}
$$

Using lemma 5. $\left(B_{3}\right)$ we find

$$
\begin{aligned}
\left\|B_{\lambda}(u, v)-B_{\lambda}\left(u_{1}, v_{1}\right)\right\| & =\sup _{\|(\xi, \eta)\| \leq 1}\left|\left\langle B_{\lambda}(u, v),(\xi, \eta)\right\rangle-\left\langle B_{\lambda}\left(u_{1}, v_{1}\right),(\xi, \eta)\right\rangle\right| \\
& =\sup _{\|(\xi, \eta)\| \leq 1}\left|b_{\lambda}((u, v),(\xi, \eta))-b_{\lambda}\left(\left(u_{1}, v_{1}\right),(\xi, \eta)\right)\right| \\
& \leq N\left\|(u, v)-\left(u_{1}, v_{1}\right)\right\|
\end{aligned}
$$

we define the operator $S: H \rightarrow H$ by

$$
S(u, v)=(u, v)-t\left(A(u, v)-B_{\lambda}(u, v)\right)
$$

where $t \in\left(0, \frac{2\left(1-\frac{\lambda\left(c_{1}+c_{2}\right)}{\lambda_{1}}\right)}{(N+M)^{2}}\right)$, The relation $(3.1)-(3.4)$ shows that for each $(u, v),\left(u_{1}, v_{1}\right) \in H$ we have

$$
\begin{gathered}
\left\|S(u, v)-S\left(u_{1}, v_{1}\right)\right\|^{2} \\
=\left\langle S(u, v)-S\left(u_{1}, v_{1}\right), S(u, v)-S\left(u_{1}, v_{1}\right)\right\rangle \\
=\left\langle(u, v)-t\left(A(u, v)-B_{\lambda}(u, v)\right)-\left(u_{1}, v_{1}\right)+t\left(A(u, v)-B_{\lambda}(u, v)\right)\right\rangle \\
=\left\|(u, v)-\left(u_{1}, v_{1}\right)\right\|^{2}-2 t\left\langle A(u, v)-A\left(u_{1}, v_{1}\right),(u, v)-\left(u_{1}, v_{1}\right)\right\rangle \\
+2 t\left\langle B_{\lambda}(u, v)-B_{\lambda}\left(u_{1}, v_{1}\right),(u, v)-\left(u_{1}, v_{1}\right)\right\rangle-2 t^{2}\langle A(u, v) \\
\left.-A\left(u_{1}, v_{1}\right), B_{\lambda}(u, v)-B_{\lambda}\left(u_{1}, v_{1}\right)\right\rangle+t^{2}\left\|A(u, v)-A\left(u_{1}, v_{1}\right)\right\|^{2} \\
+t^{2}\left\|B_{\lambda}(u, v)-B_{\lambda}\left(u_{1}, v_{1}\right)\right\|^{2} \\
\leq\left\|(u, v)-\left(u_{1}, v_{1}\right)\right\|^{2}-2 t\left\|(u, v)-\left(u_{1}, v_{1}\right)\right\|^{2}+2 t \frac{\lambda\left(c_{1}+c_{2}\right)}{\lambda_{1}}\left\|(u, v)-\left(u_{1}, v_{1}\right)\right\|^{2} \\
+2 t^{2}\left(M\left\|(u, v)-\left(u_{1}, v_{1}\right)\right\|\right)\left(N\left\|(u, v)-\left(u_{1}, v_{1}\right)\right\|\right) \\
+t^{2} M^{2}\left\|(u, v)-\left(u_{1}, v_{1}\right)\right\|^{2}+t^{2} N^{2}\left\|(u, v)-\left(u_{1}, v_{1}\right)\right\|^{2} \\
\leq\left[1-2 t\left(1-\frac{\lambda\left(c_{1}+c_{2}\right)}{\lambda_{1}}\right)+M^{2} t^{2}+N^{2} t^{2}+2 N M t^{2}\right]\left\|(u, v)-\left(u_{1}, v_{1}\right)\right\|^{2} \\
\leq \alpha\left\|(u, v)-\left(u_{1}, v_{1}\right)\right\|^{2}
\end{gathered}
$$

where

$$
\alpha=1-2\left(1-\frac{\lambda\left(c_{1}+c_{2}\right)}{\lambda_{1}}\right) t+(N+M)^{2} t^{2} \geq 0
$$

If $t=0$ or $t=\frac{2\left(1-\frac{\lambda\left(c_{1}+c_{2}\right)}{\lambda_{1}}\right)}{(N+M)^{2}}$ then $\alpha=1$.This implies that $\sqrt{\alpha}<1$ for all $t \in\left(0, \frac{2\left(1-\frac{\lambda\left(c_{1}+c_{2}\right)}{\lambda_{1}}\right)}{(N+M)^{2}}\right)$. 
Hence

$$
\left\|S(u, v)-S\left(u_{1}, v_{1}\right)\right\| \leq \sqrt{\alpha}\left\|(u, v)-\left(u_{1}, v_{1}\right)\right\|, \forall(u, v),\left(u_{1}, v_{1}\right) \in H
$$

i.e., $S$ is $\sqrt{\alpha}$ contractive with $\sqrt{\alpha}<1$. By Banach fixed point theorem (see Zeidler [10], section 1.6) it follows that there is a unique solution $(u, v) \in$ $H$

of problem $S(u, v)=(u, v)$ i.e., the problem $A(u, v)=B_{\lambda}(u, v)$ has a unique solution $(u, v) \in H$.It follows that

$$
\langle A(u, v),(\xi, \eta)\rangle=\left\langle B_{\lambda}(u, v),(\xi, \eta)\right\rangle, \forall(\xi, \eta) \in H
$$

i.e.,

$$
a((u, v),(\xi, \eta))=b_{\lambda}((u, v),(\xi, \eta)),
$$

Thus the proof of Thoerem 2 is complet.

\section{References}

[1] H. Amann, Fixed point equation and nonlinear eigenvalue problems in ordered Banach spaces, SIAM Review 18 (1976),620 709.dx.doi.org/10.1137/1018114.

[2] H. Brezis, Analyse Fonctionnelle: theorie et application, Masson, Paris, 1992.

[3] L. Collatz,Functional analysis and numerical analysis,Academic Press,New York, 1966.

[4] N. Costea, M. Mihailescu,On an eigenvalue problems involving variable exponent growth conditions, Nonlinear Anal.71 (2009),4271 4278.dx.doi.org/10.1016/j.na.2009.02.117.

[5] A. Djellit, S. Tas, Existence of solutions for a class of elliptic systems in $R^{N}$ involving the $p$-Lplacian,Electronic j. Diff. Eqns. Vol.2003 (2003), No. $56,1-8$.

[6] A. Djellit, S. Tas, On some nonlinear elliptic systems, Nonlinear Anal. 59 (2004),695 - 706.dx.doi.org/10.1016/S0362-546X(04)00279-2

[7] A. Djellit, S. Tas, Quaslinear elliptic systems with critical sobolev exponents in $R^{N}$, Nonlinear Anal. 66 (2007), $1485-1497$. 
[8] P. Drabek, N. M. Stavrakakis, N. B. Zographopoules, Multiple nonsemitrivial solutions for quasilinear systems. Differential Integral Equations 16 (12) (2003), 1519 - 1531.

[9] D. D. Hai, H. Wang, Nontrivial solutions for $p$-Lplacian systems, j. Math. Anal. Appl 330. (2007), 186 - 194.dx.doi.org/10.1016/j.jmaa.2006.07.072.

[10] Zeidler, Applied Functional Analysis: Applications in Mathematical Physics, Springer-Verlag, New York, 1995.

[11] J. Zhang, Z. Zhang, Existence results for some nonlinear elliptic systems, Nonlinear Anal. $71 \quad(2009), 2840 \quad-$ 2846.dx.doi.org/10.1016/j.na.2009.01.158. 
